\title{
Chromohysteroscopy after failed intracytoplasmic sperm injection
}

\author{
Emad E. Khalifa ${ }^{1}$, Mohamed A. EI Samra², Tamer H. Said ${ }^{2 *}$, Noha Abdin ${ }^{3}$
}

\author{
${ }^{1}$ Department of Obstetrics and Gynecology, Alexandria University, Alexandria, Egypt \\ ${ }^{2}$ Department of Obstetrics and Gynecology, Faculty of Medicine, Alexandria University, Alexandria \\ ${ }^{3}$ Ministry of Health, El Behera, Egypt
}

Received: 25 January 2016

Accepted: 01March 2016

\section{*Correspondence:}

Dr. Tamer H. Said,

E-mail: tamerhanafy74@gmail.com

Copyright: (C) the author(s), publisher and licensee Medip Academy. This is an open-access article distributed under the terms of the Creative Commons Attribution Non-Commercial License, which permits unrestricted non-commercial use, distribution, and reproduction in any medium, provided the original work is properly cited.

\begin{abstract}
Background: ICSI is the injection of a single sperm directly into the cytoplasm of the oocyte (ICSI). The failure to achieve pregnancy after ICSI has been studied over many years to reach the possible and preventable causes to avoid another failure. The aim of this study was to assess the value of using Chromohysteroscopy as a part of the work up of cases with one or more failed ICSI. The Ultimate goal is to detect the possible endometrial abnormalities and /or endometritis.

Methods: Prospective cohort study was carried out with total of 50 infertile women recruited from infertility clinic in Elshatby Maternity University Hospital, Alexandria University, Egypt. Cases were allocated in 2 groups with 25 cases in each group. The first group included cases that had a failed trial of ICSI. The second group included control cases who were seeking for fertility treatment with no previous history of ICSI or any uterine surgery or anatomical abnormalities. In the postmenstrual period, Chromohysteroscopy was done by the same surgeon and documentation of all findings in the cavity and presence of endometritis.

Results: There was a significant increase in the incidence of endometritis among the study group $68 \%$ in comparison to $16 \%$ in the control group.

Conclusions: In this study endometrial dying with methylene blue at hysteroscopy improve the detection of chronic endometritis.
\end{abstract}

Keywords: Chromohysteroscopy, Intracytoplasmic, Sperm injection

\section{INTRODUCTION}

Since the first clinical success of intra cytoplasmic perm injection (ICSI) in 1992 many centers adopted this procedure as the ultimate micro-assisted fertilization approach.

There are 2 main groups of patients that may require ICSI, patients who have a severe sperm problems which prevent them from even attempting conventional or modified form of in-vitro fertilization IVF, and patients who previously attempted IVF due to any cause of infertility but have failed to achieve fertilization. ${ }^{1,2}$ Some investigators consider implantation failure to indicate a negative pregnancy test after 2 weeks from embryo transfer. Other used the term to indicate either absence of a gestational sac on ultrasound 5 weeks after embryo transfer or of a fetal heartbeat at or beyond 5 weeks of pregnancy, and even consider failure as absence of live birth after IVF.

Many women who have uterine problems may have no problems getting pregnant, but they may have difficulty in keeping the pregnancy as they tend to miscarry (3). The evaluation of uterine factor during infertility investigations can be accomplished using ultrasonography (abdominal, transvaginal or threedimensional modality), hysterosalpingography (HSG), saline infusion sonography (SIS) and hysteroscopy. When there is a macroscopic abnormality, it is hardly 
possible to miss the diagnosis. But when there is no apparent finding, uterus is accepted as normal although endometrium still can be defective. ${ }^{4}$

Office hysteroscopy $(\mathrm{OH})$ is well-tolerated minimallyinvasive procedure, which allows reliable visual assessment of the cervical canal and uterine cavity and provide the opportunity to perform therapy in the same setting with low cost, minimal morbidity and inconvenience to the patient, and some diagnosed lesions can be operated easily using different equipment introduced through the operative channel of the hysteroscope. ${ }^{5}$ Office hysteroscopy has been proven to have superior sensitivity and specificity in evaluating the endometrial cavity. ${ }^{6}$

Chromohysteroscopy is a widely used technique in gastrointestinal imaging. Over the last decade; endoscopic systems have acquired great power due to high-resolution images. Chromohysteroscopy was first introduced after the study by Kucuk and Safali in 2008, where they combined chromo endoscopy and hysteroscopy as a new avenue to improve the diagnostic value of hysteroscopy (by using methylene blue dye) in the setting of assisted reproduction. ${ }^{7}$

Endometritis is inflammation of the endometrial lining of the uterus. In addition to the endometrim, inflammation may involve the myometrium and occasionally the parametrium. The Center for Disease Control and Prevention (CDC) 2010 sexually transmitted diseases treatment guideline defines pelvic inflammatory disease as any combination of endometritis, salpingitis, tuboovarian abscess and pelvic peritonitis. ${ }^{8}$ Endometritis is a polymicobial disease involving on average 2 to 3 organisms. In most cases it arises from ascending infection from organisms found in the normal bacterial flora. The combination of clindamycin and gentamycin administered intravenously every 8 hours has been considered the standard treatment. The combination of the second or third-generation cephalosporin with metronidazole is another popular choice. ${ }^{9}$ Marconi et al. and Kucuk and Safali reported that the endometrium is not an absorptive epithelium in normal circumstances and that structural damage of the cells allows passage of methylene blue dye into the cells. Dark blue staining represents structural damaged areas due to endometritis. $^{10,11}$

The study was designed to assess the value of using Chromohysteroscopy modality in infertility workup after failed ICSI procedures in evaluating uterine receptivity and detect any signs of chronic endometritis by a less invasive method.

\section{METHODS}

The study was conducted as a case-control study. A total of 50 infertile cases were randomly selected and allocated in 2 groups. The first group (group A), included 25 infertile women that had a failed trial of intracytoplasmic sperm injection and a second group (group B) that included 25 control cases from patient seeking for fertility treatment with no previous history of intracytpoplasmic sperm injection. The inclusion criteria were women between 20-38 years of age, nullipara or multipara, failed ICSI cycle in spite of good quality embryos in group one, time of hysteroscopy was designated between 30 and 120 days after the IVF cycle (3-4 days) postmenstrual. Women with a known or detected uterine anatomical abnormality, cancelled cycles for not having any embryos to transfer, evidence of hormonal disturbance, repeated curettage more than 3 times and/or evidence of Ashermann's syndrome were excluded from the study. All patients were subjected to detailed history taking including complete obstetric and gynecologic history, history of previous ICSI cycles, number of embryos transferred, quality of embryos, and presence of any associated pelvic pathology that was present during the ICSI cycles (hydrosalpinx, endometriosis). They underwent complete general and gynecological examination, transvaginal ultrasonography for evaluation of the uterus, ovaries and detect any pelvic pathology. All hysteroscopic operations were performed in the early follicular phase (3-4 days postmenstrual) as conventional hysteroscopy and documentation of the findings was done. Dorsal lithotomy position was used in all hysteroscopic procedure. The $2.9 \mathrm{~mm}, 30^{\circ}$ rigid telescope with an operative sheath of $5.3 \mathrm{~mm}$ was used during examination. All cases had $1 \mathrm{gm}$ ceftriaxone IV immediately before hysteroscopy. All cases had the hysteroscopy as an outpatient procedure in infertility clinic in Shatby Maternity University Hospital.

Chromohysteroscopy: in this procedure, five millimeters of $1 \%$ methylene blue dye was introduced through embryo transfer catheter (Labotect). Immediately after dye injection, the catherter was removed and introduction of the hysteroscope was done. The distension media flow started again (normal saline) and let wash of the endometrium from the dye. The uterine cavity was examined for the staining pattern. Diffuse light blue staining was considered normal. Focal, dark blue staining above the internal cervical ostium regardless of the size and number of the stained areas, will be considered positive finding. This study was approved by the institutional review board and the medical ethics committee at the Faculty of Medicine, Alexandria University, Egypt. The research complied with the international ethical research guidelines of declaration of Helsinki. An informed consent was taken from all participants after explaining the aim and concerns of the study. Data sheets were coded to ensure anonymity and confidentiality of patient's data.

\section{RESULTS}

In our study we had variable causes of infertility. In study group one had cervical factor, 6 had male factor, 5 had ovulatory problems, 3 had pelvic factor, 5 had tubal 
factor and 5 had un explained infertility while for the control group 1 had cervical factor, 9 male factor, 6 ovulatory problem 3 pelvic factor, 2 tubal factor, 4 cases of unexplained infertility.

\section{Ultrasound findings}

In the study group (A), 21 (84\%) had normal ultrasound findings, $14 \%$ has acute AVF uterus and $3(12 \%)$ had RVF uterus. In the control group (B), 22 (88\%) had normal ultrasound findings and $3(12 \%)$ had RVF uterus and there was no statistical significant difference between both groups $\mathrm{P}>0.05$.

Table 1: Comparison between the two groups as regard to ultrasound findings.

\begin{tabular}{|llllll|l|}
\hline Ultrasound & \multicolumn{3}{l}{ Study group } & \multicolumn{2}{l|}{ Control group } & P \\
\cline { 2 - 5 } findings & No. & $\%$ & No. & $\%$ & Value \\
\hline Normal & 21 & 84 & 22 & 88 & \\
\cline { 1 - 5 } Acute AVF & 1 & 4 & 0 & 0 & \multirow{2}{*}{0.600} \\
\cline { 1 - 5 } RVF & 3 & 12 & 3 & 12 & \\
\hline Total & 25 & 100 & 25 & 100 & \\
\hline
\end{tabular}

The condition of the endometrium; in the study group (A), $21(84 \%)$ out of the patient were normal), $2(8 \%)$ out of the patients ad atrophic endometrium, $14 \%$ out of the patients had thick endometrium and $14 \%$ out of the patients had thick polypoidal while in the control group $23(92 \%)$ out of the patients was normal, $1(4 \%)$ out of the patients had filmy adhesions and 1 (4\%) out of the patients was thick. There was no statistical significant difference between the two groups $\mathrm{P}>0.05$.

Table 2: Comparison between the two groups as regard to condition of the endometrium.

\begin{tabular}{|c|c|c|c|c|c|}
\hline \multirow{2}{*}{$\begin{array}{l}\text { Condition of } \\
\text { the } \\
\text { endometrium }\end{array}$} & \multicolumn{2}{|c|}{ Study group } & \multicolumn{2}{|c|}{ Control group } & \multirow{2}{*}{$\begin{array}{l}\text { P } \\
\text { Value }\end{array}$} \\
\hline & No. & $\%$ & No. & $\%$ & \\
\hline Normal & 21 & 84 & 23 & 92 & \multirow{6}{*}{0.394} \\
\hline Atrophic & 2 & 8 & 0 & 0 & \\
\hline Filmy adhesion & 0 & 0 & 1 & 4 & \\
\hline Thick & 1 & 4 & 1 & 4 & \\
\hline $\begin{array}{l}\text { Thick } \\
\text { polypoidal }\end{array}$ & 1 & 4 & 0 & 0 & \\
\hline Total & 25 & 100 & 25 & 100 & \\
\hline
\end{tabular}

Presence of endometritis: in the study group 8 (32\%) out of the patients had normal cavity (diffuse light staining) and $17(68 \%)$ had endometritis (focal dark staining) while in the control group $21(84 \%)$ had normal cavity and in 4 (16\%) had presence of endometritis. There was a high statistically significant difference between the two groups $\mathrm{P}<0.05$.
Table 3: Comparison between the two groups as regard to presence of endometritis.

\begin{tabular}{|lllllll|}
\hline $\begin{array}{l}\text { Presence of } \\
\text { endometritis }\end{array}$ & Study group & \multicolumn{2}{c|}{ Control group } & P \\
\cline { 1 - 5 } No (diffuse light) & 8 & $\%$ & No. & $\%$ & Value \\
\cline { 1 - 5 } Yes (focal dark) & 17 & 68 & 21 & 84 & \multirow{2}{*}{0.00} \\
\cline { 1 - 5 } Total & 25 & 100 & 25 & 100 & 0 \\
\hline
\end{tabular}

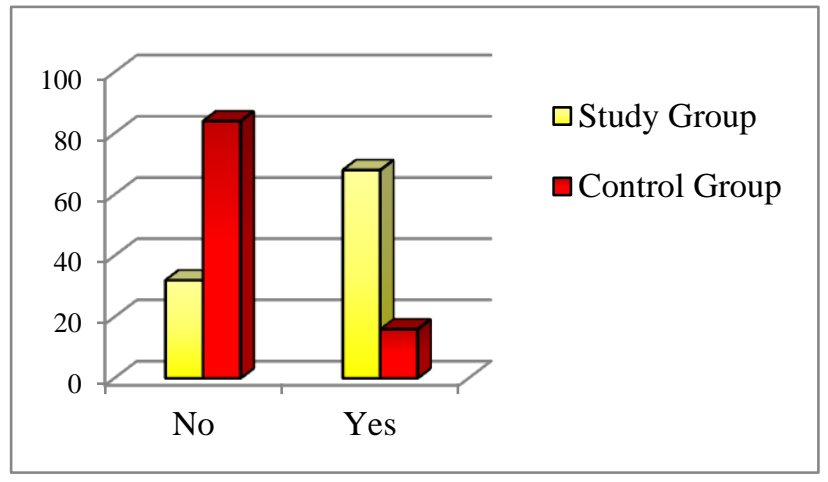

Figure 1: Comparison between the two groups as regard to presence of endometritis.

Hysteroscopic entry through the cervix: in the study group $7(28 \%)$ had difficult entry and needed uterine sound before re-entry and $18(72 \%)$ had easy entry while in the control group $5(20 \%)$ had difficult entry and 20 $(80 \%)$ had easy entry. There was no statistical significant difference between both groups $\mathrm{P}>0.05$.

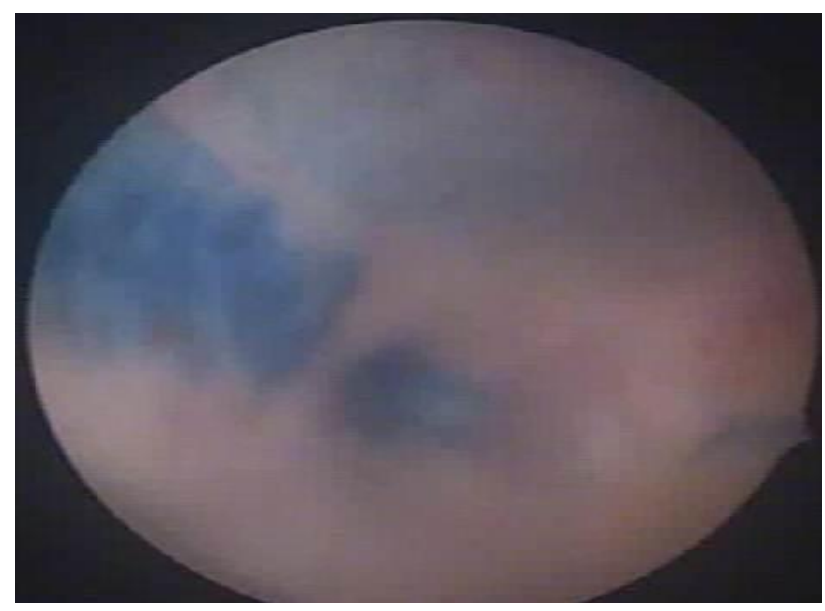

Figure 2: Hystroscopic view of dark stained areas in left lateral uterine wall and left ostea.

Day of the hysteroscopic examination in relation to the menstrual cycle: in the study group (A) the median day was $8 \pm 2$ days while in the control group the median day was $9 \pm 2$ days. No statistical significant difference between both groups $\mathrm{P}>0.05$.

The endometritis was confirmed by small biopsy using the grasper from the operative channel. The biopsy was taken from the dark stained areas. 


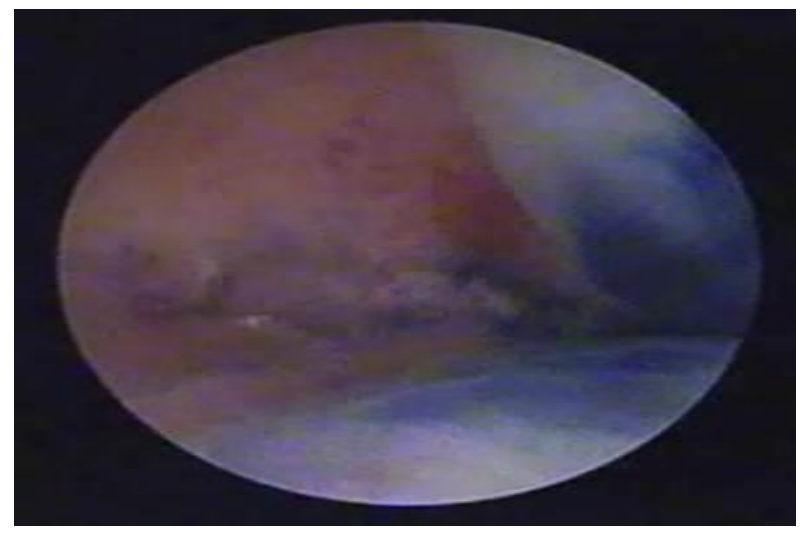

Figure 3: Hystroscopic view of dark stained areas in right ostea.

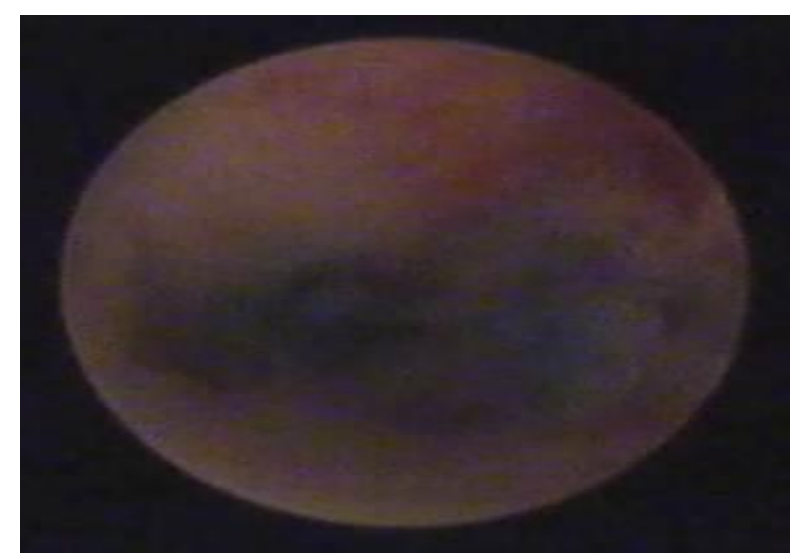

Figure 4: Hystroscopic view of dark stained areas and hyperplasia in the left ostea.

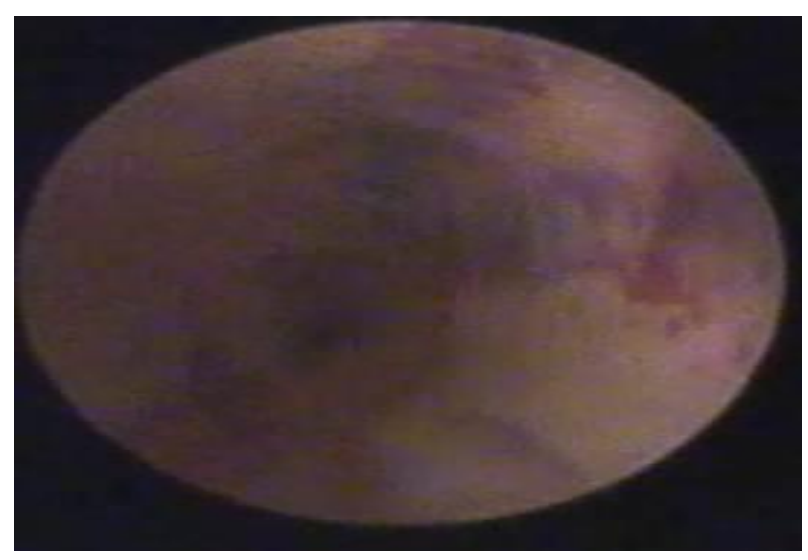

Figure 6: Hystroscopic view of light and dark stained areas in right lateral uterine wall.

\section{DISCUSSION}

Majority of papers in the current medical literature reported macroscopic endometrial pathologies like polyp, adhesion or submucous myomas in hysteroscopy after repeated IVF failure. ${ }^{12}$ When there is a macroscopic abnormality, eventually; it is detected and treated appropriately.
The corner stone in our study was assessing the cavity condition as we had in the study group 17 patients suffering from endometritis and 8 patients had normal cavity while in the control group we had 21 patients with normal cavity and only 4 patients were suffering from endometritis. These results show significant increase in the incidence of endometritis among the study group which is $68 \%$ while it is only $16 \%$ in the control group.

Regarding the ultrasound finding for the study group 21 of our patients had normal findings while one patient showed Acute AVF and 3 patients showed RVF while for the control group 22 patients showed normal result with no significant finding and we had no patients having Acute AVF and 3 patients having RVF. While regarding duration of infertility the study group showed a mean duration of 6.6 while control group showed 4.5 years.

Barati et al showed that two thirds of hysteroscopic findings were not correlated with those found on hysterography. It was shown that $54.3 \%$ of intra uterine adhesions diagnosed on hysterography were not found on direct hysteroscopic examination (false positive results). ${ }^{13}$

Zeyneloglu et al and Cicinelli et al have reported that, observations of micropolyps in hysteroscopy were a significant predictor of miscarriage after IVF-ET but they did not state an exact incidence of endometritis in their cases. $^{14,15}$

The novel technique of endometrial dying was not intended to be the sole procedure for diagnosis, but an adjunctive method to target the biopsy to correct site for an accurate diagnosis. It was shown that local endometrial defects can cause miscarriage. ${ }^{16}$ Removal of local endometrial defects may lead to replacement by healthy cells and receptivity is restored, eventually successful implantation is achieved. This theory is supported by the study of Barash et al who showed that local injury to the endometrium significantly increased the pregnancy rate in IVF. ${ }^{17}$

The presence of endometrial pathology was agreed by different studies. so hysteroscopy is highly valuable and should be applied to all such patients especially with failed ICSI. $^{18-20}$

Fatemi et al) and Karayalcin et al demonstrated that uterine cavity abnormalities in their study population were low $(11 \%$ for the 1 st one and $22.9 \%$ for the 2 nd one) while Gaviño-Gaviño et al found very high incidence of uterine pathology in their studies (64\%) with repeated IVF failure. ${ }^{21-23}$

Despite these drawbacks of non-using hysteroscopy before ICSI, many IVF clinics were reluctant in use of hysteroscopy for uterine cavity evaluation for difficulty or cost. However, office hysteroscopy with small diameter sheath (3-5 $\mathrm{mm})$, using the non-touch (vaginoscopic) technique with low pain score encouraged 
ICSI centers to start to do hysteroscopy as outpatient procedure. $^{24}$

Chronic endometritis has been related to infertility and recurrent abortion. Some authors reported that observation of micropolyps on micro hysteroscopy that was associated with $94 \%$ probability of chronic endometritis and considered it a reliable diagnostic sign. ${ }^{8}$

Hysteroscopy, with its current abilities, can never replace pathologist's eye, pathology is still the gold standard. Broader studies and/or a new vital dye can improve the diagnostic accuracy of Chromohysteroscopy, endometrial dyeing with methylene blue at hysteroscopy improves the detection of chronic endometritis. ${ }^{3}$

\section{CONCLUSION}

Chromohysteroscopy is a safe procedure which can help to improve the efficacy of hysteroscopy and to increase the success rate of ICSI. It is highly recommended to do hysteroscopy in cases of repeated ICSI failure. This simple procedure could be a valuable screening tool to detect endometritis and other subtle endometrial pathology not detected in different imaging techniques.

Funding: Not required

Conflict of interest: None declared

Ethical approval: The study was approved by the Institutional Ethics Committee

\section{REFERENCES}

1. Oehninger S, Gosden RG. Should ICSI be the treatment of choice for all cases of in-vitro conception? No, not in light of the scientific data. Hum. Reprod. 2002;17:2237-42.

2. French DB, Sabanegh ES, Goldfarb J, Desai N. Does severe teratozoospermia affect blastocyst formation, live birth rate, and other clinical outcome parameters in ICSI cycles? Fertil Steril. 2010;93(4):1097-103.

3. Petrozza JC. Assisted Reproduction Technology Hum Reprod Update. 2006:1039-1049.

4. Oliveira FG, Abdelmassih VG, Diamond MP, Dozortsev D, Nagy ZP, Abdelmassih R. Uterine cavity findings and hysteroscopic interventions in patients undergoing in vitro fertilization-embryo transfer who repeatedly cannot conceive. Fertil Steril. 2003;80:1371-5.

5. Kermer C, Duffy S, Moroney M. Patient's satisfaction with outpatient hysteroscopy versus day care hysteroscopy: Randomized controlled trial. $\mathrm{Br}$ Med J. 2000;320:279-82.

6. Workowski KA, Berman S. Sexually transmitted diseases treatment guidelines, 2010. MMWR Recommend Rep. 2010;59:1-110.

7. Marconi G, Vilela M, Quintana R, Diradourian M, Young E, Sueldo C. New observations on endometrial physiology after Transcervical injection of methylene blue dye. Fertil Steril. 2004;82:1700-4.

8. Gardner DK, Schoolcraft WB, Wagley L, Schlenker $\mathrm{T}$, Stevens J and Hesla J. A prospective randomized trial of blastocyst culture and transfer in in-vitro fertilization. Hum. Reprod. 1998;13:3434-40.

9. French LM, Smaill FM. Antibiotic regimens for endometritis after delivery. Cochrane Database Syst Rev. 2004;CD001067.

10. Marconi G, Vilela M, Bello J. Sueldo Endometrial lesions caused by catheter used for embryo transfers: a preliminary report Fertil Steril. 2003;80:363-7.

11. Kucuk T, Safali M. Chromohysteroscopy for evaluation of endometrium in recurrent in vitro fertilization failure $\mathrm{J}$ Assist Reprod Genet. 2008;25:79-82.

12. Oliveira FG, Abdelmassih VG, Diamond MP, Dozortsev D, Nagy ZP, Abdelmassih R. Uterine cavity findings and hysteroscopic interventions in patients undergoing in vitro fertilization-embryo transfer who repeatedly cannot conceive. Fertil Steril. 2003;80:1371-5.

13. Barati M, Zargar M, Masihi S, Borzoo L, Cheraghian B. Office hysteroscopy in infertility. International journal of fertility and sterility. 2009;3(1):17-20.

14. Zeyneloglu HB, Onalan G, Dursun P, Altuntas B. Polypoid appearance of the endometrium on office hysteroscopy a significant predictor of miscarriage after IVF-ET. Fertil Steril. 2007;88:S96.

15. Cicinelli E, Resta L, Nicoletti R, Zappimbulso V, Tartagni M, Saliani N. Endometrial micropolyps at fluid hysteroscopy suggest the existence of chronic endometritis. Hum Reprod. 2005;20:1386-9.

16. Bajekal N, Li TC. Fibroids, infertility and pregnancy wastage. Hum Reprod Update. 2000;6:614-20.

17. Barash A, Dekel N, Fieldust S, Segal I, Schechtman E, Granot I. Local injury to the endometrium doubles the incidence of successful pregnancies in patients undergoing in vitro fertilization. Fertil Steril. 2003;79:1317-22.

18. National Institute for Clinical Excellence Guidelines tools to optimize the IVF-ET procedure and its cost effectiveness. London: RCOG Press; 2004.

19. Wkim R, Raneiri DM. Assessment of the uterine cavity by saline hysteron-contrast-sonography before IVF: presented at the XVIIIth annual meeting of the ESHRE, Vienna, Austria Hum Reprod Program Suppl. 2002;17:165-6.

20. Shokeir TA, Shalan HM. Significance of endometrial polyps detected hysteroscopically in amenorrheic infertile women J Obstet Gynecol Res. 2004;30:84-9.

21. Fatemi HM, Kasius JC, Timmermans A. Prevalence of unsuspected uterine cavity abnormalities diagnosed by office hysteroscopy prior to in vitro fertilization Hum Reprod. 2010;25:1959-65.

22. Karayalcin R, Ozcan S, Moraloglu O. Results of 2500 office-based diagnostic hysteroscopies before IVF Reprod Biomed Online. 2010;20:689-93.

23. Gaviño-Gaviño F, Guzmán-González E, ReyesMuñoz E, J. Villalpando-Bravo. Impact of office hysteroscopy in patients with a history of two or 
more cycles of IVF-ET failed pre-ICSI in assisted reproduction center Gynecol Obstet Mex. 2010;78:914.

24. Lorusso F, Ceci O, Bettocchi S. Office hysteroscopy in an in vitro fertilization program Gynecol Endocrinol. 2008;24:465-9.
Cite this article as: Khalifa EE, El Samra MA, Said TH, Abdin N. Chromohysteroscopy after failed intracytoplasmic sperm injection. Int J Reprod Contracept Obstet Gynecol 2016;5:1065-70. 